\title{
TV-RING and ImmersiaTV: Present and Future of Television
}

\author{
Marc Aguilar, Pau Pamplona, Sergi Fernández \\ Media Internet Area, i2CAT Foundation \\ Gran Capità 2-4, Nexus I building, 203; 08034 Barcelona, Spain \\ marc.aguilardi2cat.net, pau.pamplona@i2cat.net, \\ sergi.fernandezei2cat.net
}

\begin{abstract}
In this paper we present our results from a research project on multicamera HbbTV applications, the TV-RING project, and situate these results as part of a broader push towards the development of innovative media experiences for TV audiences. The main lessons learned in the framework of this project are outlined, with special attention paid to principles of usability, insights on the user experience, and recommendations for the selection of multicamera content. These results were generated as the output of a usercentered design and experimentation process, involving requirements elicitation, iterative prototyping and large-scale live pilots. Finally, we sketch how immersive media can address current issues as regards the user experience raised by TV-RING, and introduce related ongoing work in the ImmersiaTV project on broadcast omnidirectional video.
\end{abstract}

Keywords. Connected TV, HbbTV, User Evaluation, Multicamera, Immersive TV

\section{Introduction}

In the last years, the mission of i2CAT Foundation's Media Internet Area has been centered on the development of innovative audiovisual experiences. In the framework of the TV-RING project, the focus has been placed on the user-centered development of adaptive-streaming, MPEG-DASH-enabled multicamera HbbTV applications. In the upcoming ImmersiaTV project, the focus of work will shift to immersive television, an emerging paradigm which holds the promise to revolutionize the TV viewing experience. This position paper presents an overview of the main outcomes of the TV-RING project's multicamera pilot, and attempts to delineate the relevance and continuity of its research in the ImmersiaTV project.

\section{The TV-RING project multicamera pilot}

The multicamera pilot application, developed by TV-RING partner Televisió de Catalunya (TVC), consists of two main scenarios or areas of work. Firstly, a VoD service 
of content that can be accessed interactively from one or two different points of view or 'renditions' and secondly, accessing multiple renditions on a live TV show. Content is accessed using MPEG-DASH in all cases, allowing for seamless and instantaneous timeline shifting in the first case and actual live content in the second case. All contents are offered in three different qualities, ranging from the 7Mbps at $\mathrm{HD}$, to an intermediate $5 \mathrm{Mbps}$, to $3 \mathrm{Mbps}$ at SD using MPEG- DASH.

The application is made up of different screens. It is mainly composed of a screen with two lists of contents, one with a live content and the other with the VOD content. The user can interact with the application using the remote control of the HbbTVenabled TV set device, enabling the user to select content, control video playback and specify video renditions. Finally, in the start menu, the user has option to display help screen by pressing the blue button on the remote control. Based on insights from the preliminary user evaluation, the application was enhanced with a second screen functionality to allow users to control the HbbTV application using a mobile device as remote control. In the following figure (Fig. 1), a workflow of the application can be found to better describe the different GUI interactions. For further details on the test application, readers are kindly referred to the bibliography $[1,2,3]$.

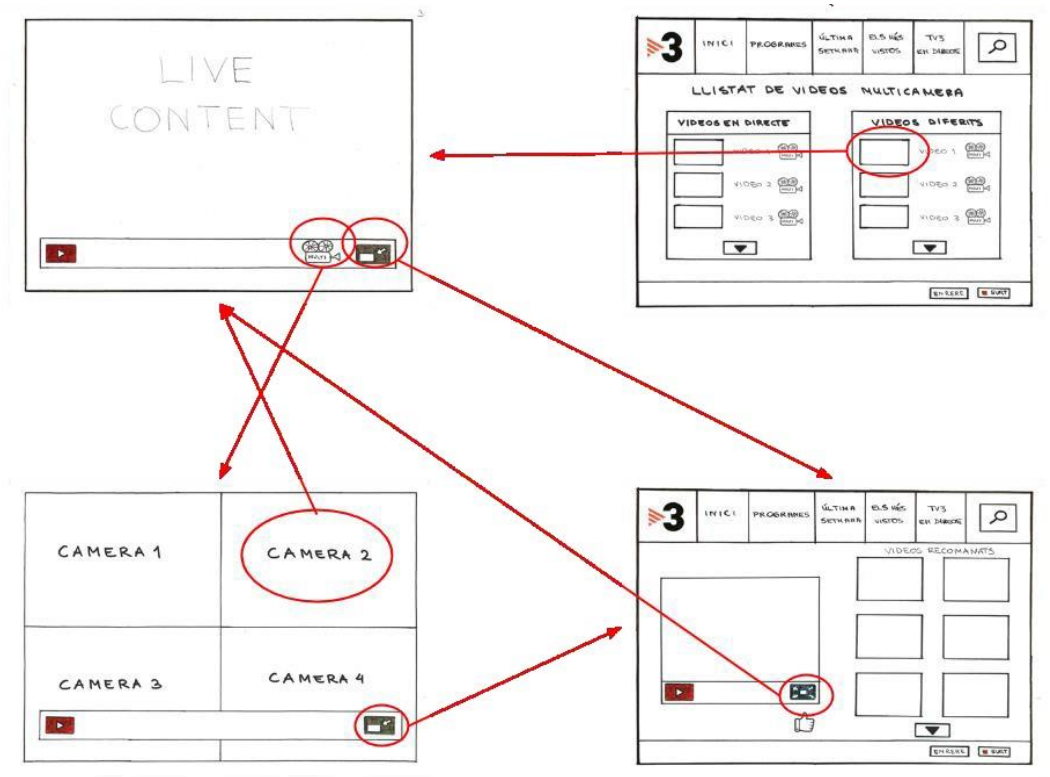

Fig. 1. Multicamera pilot application basic interaction pattern

As delineated in the piloting plan, a sequence of tests was carried out over a thirteenmonth period [4]. This constituted the final phase of a user-centered design process which had previously involved users in two rounds of requirements analysis and three iterations of prototype refinement. First, four small-sample controlled pilots were 
executed from December 2014 to June 2015. This phase involved twenty households in the town of Gurb, in central Catalonia. Each household was given an HbbTV 1.5 device capable to play MPEG-DASH and last generation services. Test users were connected to a monitored fibre network of $100 \mathrm{Mb} / \mathrm{s}$ using a local controlled CDN, from which detailed analytics could be monitored. At this stage, the goal was to finetune the usability of the test application, sort out any outstanding technical issues, and obtain market data on the most attractive contents for users to be used in larger experiments. The contents for these tests were a singing contest show, a special news report on the Spanish local elections, and two football matches.

This first phase of controlled-sample pilot tests set the stage for the two large-scale open pilots. A preparatory pilot was carried out in September, with news coverage of the Catalan national day rally, to ensure the successful deployment of the envisioned live pilots. The selected contents for the live pilots were two FC Barcelona football matches in September and November 2015. These open live pilots were followed by an audience of about 4000 and 6200 TV devices which accessed the project's HbbTV application (see Fig. 2), representing an audience of thousands of users interacting with the offered multicamera services. Extensive data was obtained on the patterns of usage of the application, technical performance parameters and several metrics of user satisfaction. An in-depth analysis of these data has yielded rich information on HbbTV market penetration potential, models of user segmentation and clustering, and analytics on the user's behavior in real-life settings.
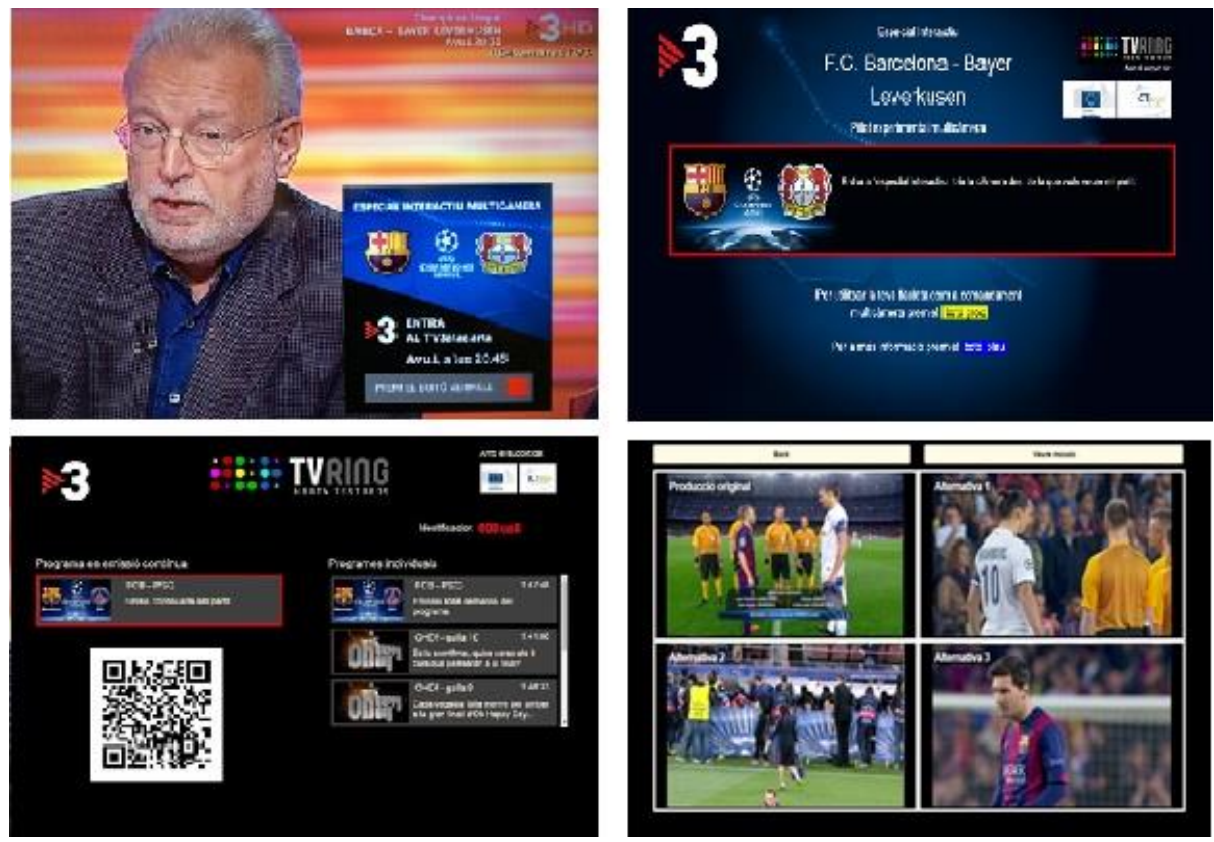

Fig. 2. Screenshots of application during open live pilot tests 
Finally, three batteries of in-lab technical tests were carried out as well in November and December 2015. These tests were designed to test the performance of the TVC application across a range of TV devices, to evaluate the performance of the MPEGDASH encoder, and to determine experimentally the impact of hardware performance on latency and user satisfaction.

\section{Lessons learned}

Multicamera content can be attractive for audiences under certain usability conditions and for certain programs. Nevertheless, there are constraints from a user-centric point of view that may limit its generalization if not properly addressed by HbbTV application designers.

Early on during the user evaluation activities, it was found that traditional remote controls offer very poor usability for users accustomed to more agile handheld device navigations. Several approaches to replace or complement the traditional remote control have been successfully implemented, such as speech and gesture recognition controls [5]. In the TV-RING project multicamera pilot, a Second Screen solution was tested on field trials. The results obtained give weight to the hypothesis that a Second Screen solution can overcome the app navigation limitations posed by conventional remote controls. However, there are some challenges to the uptake of such solutions, as the less technically-savvy users (which usually coincide with older cohorts) are held back by the lack of compatible handhelds in some households and the need to link the devices. Easing the Second Screen - TV linking process, for example with QR codes and visual step-by-step instructions, is paramount to accelerate uptake.

A number of general usability recommendations for the design of HbbTV applications emerge from the prototype refinement and live piloting phases. These include offering very agile navigations, ensuring consistency in commands by using color codes (i.e. red means go back, green means go forward), making explicit to the user the function of every button (forward, back to main screen, exit app), limiting clutter in the screen with minimalistic designs so that content is always the center of attention, and displaying a machine reaction for every user action [6].

A significant finding is that hardware performance problems have a serious impact on the user experience. Users may display some patience with waiting while loading contents and slight degradations in video quality, but are not so understanding with instances in which they feel their TVs take an excessive amount of time to process their requests. More specifically, hardware delays above the 5-6 second threshold were found to produce frustration in most test users. This frustration increases progressively as delays become longer, quickly deteriorating the user experience. Delays above the 8-10 second mark were considered not acceptable by all test users. Performance problems attributable to hardware are very difficult to address by app developers. Nevertheless, it has been found that their negative impact on the user experience 
can be minimized by the simple expedient of adding any indication of "task in progress" for the user (i.e. a completion bar, a "wait..." sign), and this reassures the user and may compel her to "stay tuned" [7].

Content selection is critical for the success of a multicamera HbbTV application. Programs in which the relevant action may happen simultaneously in several locations are the best picks for multicamera content. Sports such as football, basketball or tennis, and racing events like the Formula One or MotoGP competitions have been identified as particularly suitable contents for multicamera. Other kinds of contents such as special informative events (i.e. demonstrations, election days) and song contests were piloted during the course of the TV-RING project. The audience's reaction to these programs was fairly positive as well. Nevertheless, a lesser level of interest was detected, as many users did not see the value of multicamera services for those kinds of programs vis-à-vis the broadcast content produced by an experienced audiovisual producer.

\section{Beyond the current user experience: the ImmersiaTV project}

Immersive TV has the potential to overcome these limitations by bringing to audiences a radically novel experience. The majority of European TV consumers already watch TV programs in a multi-display environment. Second screens - mostly smartphones, tablets or laptops - are generally used to check information not directly related to the events in the TV content being watched. As a result, the attention of the audience is generally divided between these different streams of information. Broadcasters have tried (like in TV-RING) to orchestrate all these different rendering platforms to complement each other consistently. However, their success is limited, and this is due, at least in part, to the very different formats in which information is delivered (web-based texts, mobile apps, hybrid apps, traditional broadcast television, etc.).

The ImmersiaTV project will create new forms of digital storytelling and broadcast production that, by putting omnidirectional video at the center of the creation, production and distribution of broadcast content, delivers an all-encompassing experience that integrates the specificities of immersive displays, and the feeling of "being there", within the contemporary living room. We propose a new form of broadcast omnidirectional video that offers end-users a coherent audiovisual experience across head mounted displays, second screens and the traditional TV set, instead of having their attention divided across them. This new experience will seamlessly integrate with and further augment traditional TV and second screen consumer habits. In other terms: the audience will still be able to watch TV sitting on their couch, or tweet comments about it. However, by putting omnidirectional content at the center of the creation, production and distribution processes, the audience will also be able to use immersive displays to feel like being inside the audiovisual stream. 


\section{Conclusions}

This paper has discussed the work performed in the framework of the TV-RING project in the field of multicamera HbbTV applications, and outlined the main conclusions learned as regards the user's preferences, experience and behavior with such services. Multicamera services have been well received by audiences, provided that these are offered via user-friendly apps, run on well-performing devices, and supported by adequate contents. These lessons will inform the work to be done in the ImmersiaTV project. In this Horizon 2020 initiative, which started January 2016, an attempt is being made to redefine TV viewing by bringing an immersive experience to the user's household.

\section{Acknowledgements}

The research for this paper has been partially funded by the CIP-PSP Programme under grant agreement 325209 .

\section{References}

1. Vogl, A. et al: D3.3.1 Service Implementation Report. Public report, TV-RING project (2014). http://www.TV-RING.eu/documentation/deliverables/

2. Baumann, F. et al: D3.3.2. Services and Applications. Public report, TV-RING project (2014)

3. Pujals, D. et al: D3.4 Description of Technical Pilot Infrastructures. Public report, TVRING project (2015)

4. Pamplona, P. et al: D4.2. Pilot Execution Report. Public report, TV-RING project (2015)

5. Vanattenhoven, J., Geerts, D., De Grooff, D.: Television Experience Insights from HbbTV. In: Proceedings of 2nd International Workshop on Interactive Content Consumption at TVX 2014 (pp. 32-34). Newcastle, UK (2014)

6. Glaser, S. et al: D3.2 Intermediate Evaluation Report. Public report, TV-RING project (2014)

7. Vanattenhoven, J. et al: D4.3. Evaluation Results. Public report, TV-RING project (2016) 